\title{
Numerical benchmark campaign of COST Action TU1404 - microstructural modelling
}

\author{
Mateusz Wyrzykowski ${ }^{1 *}$, Julien Sanahuja ${ }^{2}$, Laurent Charpin ${ }^{2}$, Markus Königsberger ${ }^{3}$, Christian Hellmich ${ }^{3}$, \\ Bernhard Pichler ${ }^{3}$, Luca Valentini ${ }^{4}$, Túlio Honório ${ }^{5}$, Vit Smilauer ${ }^{6}$, Karolina Hajkova ${ }^{6}$, Guang Ye, Peng \\ $\mathrm{Gao}^{7}$, Cyrille Dunant $^{8}$, Adrien Hilaire ${ }^{9}$, Shashank Bishnoi ${ }^{10}$, Miguel Azenha ${ }^{11}$
}

\author{
Empa, Swiss Federal Laboratories for Materials Science and Technology, Switzerland \\ EDF, R\&D MMC, France \\ TU Wien, Austria \\ University of Padua, Italy \\ Université Paris-Est, Laboratoire Navier (UMR 8205), CNRS, ENPC, IFSTTAR, France \\ Czech Technical University in Prague, Czech Republic \\ TU Delft, The Netherlands \\ Department of Engineering, University of Cambridge, UK \\ EPFL, Lausanne, Switzerland \\ IIT Delhi, India \\ ISISE, University of Minho, Portugal
}

Received: 5 December 2017 / Accepted: 25 December 2017 / Published online: 30 December 2017

(C) The Author(s) 2017. This article is published with open access and licensed under a Creative Commons Attribution 4.0 International License.

\begin{abstract}
This paper presents the results of the numerical benchmark campaign on modelling of hydration and microstructure development of cementitious materials. This numerical benchmark was performed in the scope of COST Action TU1404 "Towards the next generation of standards for service life of cement-based materials and structures". Seven modelling groups took part in the campaign applying different models for prediction of mechanical properties (elastic moduli or compressive strength) in cement pastes and mortars. The simulations were based on published experimental data. The experimental data (both input and results used for validation) were open to the participants. The purpose of the benchmark campaign was to identify the needs of different models in terms of input experimental data, verify predictive potential of the models and finally to provide reference cases for new models in the future. The results of the benchmark show that a relatively high scatter in the predictions can arise between different models, in particular at early ages (e.g. elastic Young's modulus predicted at $1 \mathrm{~d}$ in the range $6-20 \mathrm{GPa}$ ), while it reduces at later age, providing relatively good agreement with experimental data. Even though the input data was based on a single experimental dataset, the large differences between the results of the different models were found to be caused by distinct assumed properties for the individual phases at the microstructural level, mainly because of the scatter in the nanoindentation-derived properties of the C-S-H phase.
\end{abstract}

Keywords: Benchmark; Numerical modelling; Microstructure; COST TU1404

\section{Introduction}

The campaign reported here has been performed within the activities of the COST Action TU1404 "Towards the next generation of standards for service life of cement-based materials and structures" [1]. The main objective of the Action is to contribute to the development of a new generation of guidelines/recommendations to predict/evaluate the service life of cement-based materials and structures in Europe by integrating the most recent developments in experimental and numerical approaches, with particular focus on concrete performance at early ages. One of the major tools for reaching this objective is by means of developing and validating numerical models that allow one to predict the evolution of material properties and eventually their service life performance in a real structure. To this end, a series of numerical benchmarking campaigns is carried out within the Action. These benchmarking campaigns have the following objectives:

- Validate the numerical models by means of experimental data, to be used both for the models currently taking part in the campaign and for future verification or validation of the new models;

* Corresponding author: Mateusz Wyrzykowski, Tel. +41 58765 45 41, E-mail: mateusz.wyrzykowski@empa.ch 
- Identify the needs of the models in terms of input data to better assist the design of experimental campaigns targeted for characterization (performed as within the scope of COST Action TU1404, particularly under the designation of 'Extended Round Robin Testing Program' $\mathrm{RRT}+$ );

- Present the capabilities and limitations of the models, in particular to the experimentalists from outside the modelling community;

- Serve as a platform for discussing and exchanging experience in the modelling community.

This paper deals with the first stage of the benchmarking campaign with input experimental data made fully open to the participants. This stage was planned as a preliminary action before launching the broader benchmarking campaign devoted to validation of the models with the experimental data obtained in the 'Extended Round Robin Testing Program' RRT+ [2].

The simulations focus on hydration and microstructure development of cement paste and mortar and corresponding evolution of the mechanical properties.

The call for benchmark participation was announced in March 2016 and the results were submitted by the participants by September 2017. Some of the results were presented in a form of oral presentations at two conferences: the MSSCE 2016 conference in Denmark on 23 August 2016 and at the CONCRACK5 conference in Tokyo on 25 April 2017. This publication presents a comprehensive description of the input data and modelling results along with a critical discussion of the modelling tools and their predicting capabilities.

\section{Experimental data used in the benchmark}

The experimental dataset used in this benchmark was mostly obtained in previous works [3, 4]. Some additional tests have been performed directly on the same batch of materials as in $[3,4]$ in order to provide necessary input for the specific models.

The experiments were performed on cement paste at a water-to-cement ratio by mass $(w / c)$ of 0.30 . The cement used was CEM I $42.5 \mathrm{~N}$ with a Blaine fineness of $2.91 \mathrm{~m}^{2} / \mathrm{g}$, specific surface (determined with laser diffraction) of 1.53 $\mathrm{m}^{2} / \mathrm{g}$ and density of $3.09 \mathrm{~g} / \mathrm{cm}^{3}$. Oxide composition determined with XRF was (by mass): $\mathrm{CaO} 63 \%$ (free $\mathrm{CaO}$ $0.52 \%, \mathrm{CaO}$ in calcite $2.32 \%$ ), $\mathrm{SiO}_{2} 20 \%, \mathrm{Al}_{2} \mathrm{O}_{3} 4.5 \%, \mathrm{Fe}_{2} \mathrm{O}_{3}$ $2.9 \%, \mathrm{MgO} 1.9 \%, \mathrm{SO}_{3} 2.8 \%, \mathrm{~K}_{2} \mathrm{O} 0.95 \%, \mathrm{Na}_{2} \mathrm{O} 0.19 \%$. This yielded the following modified Bogue composition $\left(\mathrm{SO}_{3}\right.$ in clinker assumed as 0.5\%) [5]: $\mathrm{C}_{3} \mathrm{~S} 60.8 \%, \mathrm{C}_{2} \mathrm{~S} 12.5 \%, \mathrm{C}_{3} \mathrm{~A}$ $4.3 \%, \mathrm{C}_{4} \mathrm{AF} 9.9 \%$. The measured particle size distribution of the cement is presented in Fig. 1.

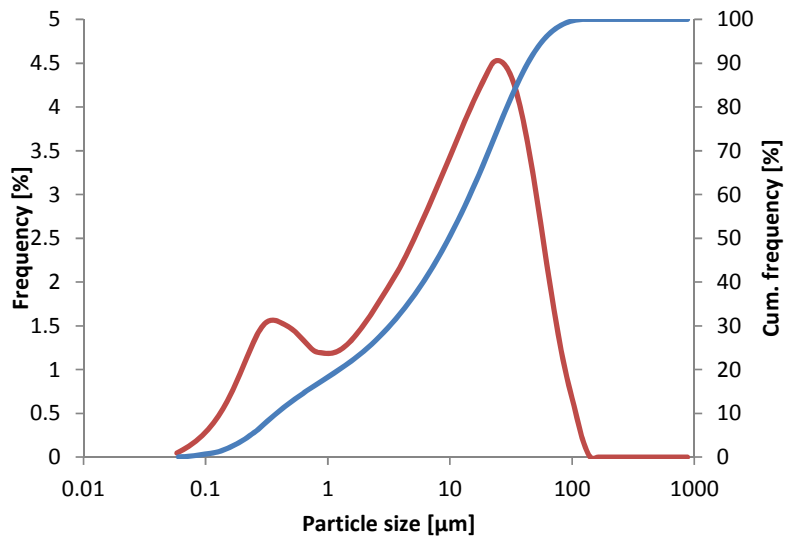

Figure 1. Particle size distribution of the cement used (laser diffraction).

The pastes were mixed in a vacuum mixer in order to minimize the effect of entrapped air voids. The mixes were prepared using deionized water and polycarboxylate-based superplasticizer ( $0.5 \%$ by mass of cement).

The samples were cured in moisture-sealed conditions at a temperature of $20 \pm 0.5^{\circ} \mathrm{C}$.

The basic property of the mixes used in this benchmark was evolution of hydration heat release, as shown in Fig. 2 . It was measured with isothermal calorimetry on duplicate samples. Cement pastes were mixed externally and about $6 \mathrm{~g}$ of cement paste were used for a specimen. The measurements started $1 \mathrm{~h}$ from mixing. The hydration heat data can be used for estimation of the hydration degree after estimating the heat released at complete hydration (the value of $454 \mathrm{~J} / \mathrm{g}$ was estimated based on the phase composition of cement in [3]).

Among different properties measured on cement paste in [4], the evolution of elastic properties and compressive strength were used for the benchmark. Mechanical properties were measured on mixtures prepared in a vacuum mixer. Elastic Young's modulus was measured with a static test in compression (loading to $30 \%$ of compressive strength) on triplicate samples $\left(25 \times 25 \times 100 \mathrm{~mm}^{3}\right.$ prisms). Dynamic elastic Young's modulus and shear modulus were also measured using resonant ultrasound spectroscopy on triplicate $25 \times 25 \times 100 \mathrm{~mm}^{3}$ prisms. The results of E-modulus (static and dynamic) as well as the shear modulus are shown in Fig. 3, where the error bars represent the standard deviation (note very low scatter). Compressive strength was measured on single prismatic $25 \times 25 \times 100 \mathrm{~mm}^{3}$ samples (two halves measured per strength at each age). The corresponding results are shown in Fig. 4.

\section{$3 \quad$ Models applied}

Seven groups applying different models of cement-based materials participated in the benchmark, see Table 1 . The models are briefly presented in the following section. The detailed input data used in the models is summarized in the supplementary materials [6]. 


\begin{tabular}{|c|c|c|c|c|}
\hline No. & Model & Investigators & Institution & Predicted results* \\
\hline 1 & $\begin{array}{l}\mu \mathrm{ic} \\
\text { and AMIE }\end{array}$ & $\begin{array}{l}\text { Cyrille Dunant }^{1}, \\
\text { Adrien Hilaire }^{1}, \\
\text { Shashank Bishnoi }^{2}\end{array}$ & $\begin{array}{l}\text { 1-EPFL, Switzerland } \\
\text { 2-IIT Delhi, India }\end{array}$ & Hydration heat, elastic properties \\
\hline 2 & $\mathrm{Vi}(\mathrm{CA}) 2 \mathrm{~T}$ v2 & $\begin{array}{l}\text { Julien Sanahuja } \\
\text { Laurent Charpin }\end{array}$ & EDF, France & Hydration heat, elastic properties \\
\hline 3 & $\begin{array}{l}\text { Micromechanical } \\
\text { homogenization }\end{array}$ & $\begin{array}{l}\text { Markus Königsberger } \\
\text { Christian Hellmich } \\
\text { Bernhard Pichler }\end{array}$ & TU Wien, Austria & Compressive strength \\
\hline 4 & $\begin{array}{l}\text { Micromechanical } \\
\text { analytical model }\end{array}$ & Tulio Honorio & $\begin{array}{l}\text { Université Paris-Est, } \\
\text { Laboratoire Navier (UMR } \\
\text { 8205), CNRS, ENPC, IFSTTAR, } \\
\text { France }\end{array}$ & $\begin{array}{ll}\text { Hydration heat, elastic properties, } \\
\text { thermal expansion, autogenous } \\
\text { shrinkage }\end{array}$ \\
\hline 5 & VCCTL & Luca Valentini & University of Padua, Italy & Elastic properties \\
\hline 6 & $\begin{array}{l}\text { Micromechanical } \\
\text { numerical model }\end{array}$ & $\begin{array}{l}\text { Vit Smilauer } \\
\text { Karolina Hajkova }\end{array}$ & $\begin{array}{l}\text { Czech University in Prague, } \\
\text { Czech Republic }\end{array}$ & $\begin{array}{l}\text { Hydration heat, elastic properties, } \\
\text { compressive strength }\end{array}$ \\
\hline 7 & HYMOSTRUC3D & $\begin{array}{l}\text { Guang Ye } \\
\text { Peng Gao }\end{array}$ & TU Delft, The Netherlands & $\begin{array}{l}\text { Degree of hydration, porosity, elastic } \\
\text { properties, compressive strength, } \\
\text { autogenous shrinkage, pore solution } \\
\text { chemistry }\end{array}$ \\
\hline
\end{tabular}

* only hydration evolution, elastic properties and compressive strength are presented here

\section{1 $\mu i c$ and AMIE}

$\mu$ ic is a modelling platform that uses the vector approach for the description of cement and evolving microstructure [7]. The benefit of the vector approach is that the model has no limits of resolution. The microstructure is modelled as a periodic volume with cement particles defined as spheres. $\mu \mathrm{ic}$ is a general particulate reaction model that can be used to model any type of cement, as long as its chemistry is understood, or chemical data is available. In the current case, the hydration of Portland cement has been modelled, with each cement phase being treated separately. All particles formed are assumed to be spherical due to computational limitations. $\mathrm{CH}$ is formed from the hydration of alite and belite, based on hydration stoichiometry. Aluminate first hydrates with gypsum to form ettringite, which later converts to monosulphate, consuming some more aluminate and water. $\mu$ ic does not define kinetics: it has plugins which can be changed depending on the goal of the simulation. In this study, two sets of kinetic plugins were used. The first one forces phase evolution. It assumes that C$\mathrm{S}-\mathrm{H}$ fills very rapidly all the available porosity ( $\mathrm{C}-\mathrm{S}-\mathrm{H}$ diffuse filling). The second variant accounts explicitly for the dissolution and precipitation of phases. Unlike the first one, it assumes the $\mathrm{C}-\mathrm{S}-\mathrm{H}$ is denser and grows slower from the original surface of the grains (C-S-H growing). This produces two heat evolutions and two different microstructures. These two variants yield similar results, although the diffuse filling was developed for the purpose of modelling the evolution of mechanical properties, and the growing was focused on obtaining accurate kinetics.

Next, the obtained microstructures were used for simulating the elastic Young's modulus. The same model of phase evolution was used in both set of generated microstructures. This was done using Automated Mechanics for Integrated Experiments (AMIE) [8, 9]. AMIE is a FEM code for microstructural simulations. Based on the assumed values of stiffness of the individual hydration products and of unhydrated cement the stiffness of the microstructure is calculated from the stress and strain obtained when the generated microstructure is loaded. The simulations use a homogenous displacement boundary condition and calculate the strain from the averaged deformation over the volume. This yields an upper bound of the stiffness. No postprocessing was applied, therefore the mesh contains overestimated contact in the first steps of hydration due to the following artifact. When the meshing of microstructures is carried out, two neighbouring voxels, which do not contain particles that are connected to each other, can be marked as solid. Since neighbouring voxels share nodes, they then appear to be connected, leading to the stiffness of the microstrcuture to appear to be higher than it actually is [10]. Although the application of the so-called "burning algorithms" can reduce such meshing artefacts, this has not been used here so as to have a more uniform comparison between models.

\subsection{Vi(CA)2T v2}

Virtual Cement and Concrete Ageing Analysis Toolbox (Vi(CA)2T V2) is based on the concept of a "virtual lab" or "virtual material": from the mix design details, it is able to estimate physical properties (such as the evolution of hydration heat, capillary porosity or water content) and mechanical behaviors (such as the evolution of the Young's modulus, or basic creep). To do so, it embeds two kinds of models:

- Cement paste hydration models: combining chemistry and kinetics, from the initial mix design information (including clinker composition), the amounts of phases (different anhydrous phases, various hydrates, water and porosity) and the hydration macroscopic properties (temperature, released heat) are estimated as a function of time.

- Micromechanical models: taking advantage of upscaling techniques, from the amount and elementary behavior of phases, the effective mechanical behavior is estimated 
(such as stiffness or some characteristics of basic creep). The phases considered in the model were: anhydrous phases, hydrated phases (split into inner and outer) and porosity.

For more details on $\mathrm{Vi}(\mathrm{CA}) 2 \mathrm{~T}$ V2, the industrial needs, the currently implemented models, challenges raised by development of such a tool, and validation, see [11-13]. Models currently implemented only allow one to estimate the cumulative heat release and the Young's modulus, among the results proposed to benchmark. It is worth noting that the model was applied without fitting any parameters.

\subsection{Micromechanical homogenization}

The micromechanical homogenization approach of Pichler and Hellmich [14] and Pichler et al. [15] was applied to upscale the microscopic strength of needle-shaped hydration products to the macroscopic strength evolution of cement pastes. In this context, a two-scale micromechanics representation was used:

- Cement paste is modeled as a matrix-inclusion composite, with spherical clinker grains embedded in a hydrate foam matrix (modeled by means of the MoriTanaka scheme);

- The hydrate foam matrix is considered to exhibit a polycrystalline microstructure which is built up of spherical capillary porosity and of needle-shaped hydrate phases oriented uniformly in all spatial directions (modeled by means of the self-consistent scheme).

The basic idea standing behind micro-to-macro upscaling of quasi-brittle strength is that the macroscopic strength of cement paste is reached once microscopic stress "peaks" in the hydrate phases reach the hydrate strength. Microscopic stress peaks are quantified based on quadratic deviatoric stress averages [14, 15]. As for mechanical phase properties, the key quantity governing all model predictions is the deviatoric hydrate strength which has been identified as 70 MPa based on nanoindentation test results from [16], see also [15]. Elastic phase properties are taken from [14]. As for phase volume evolutions, the simulations rely on the Powers' model, see [14]. In this context, only two input parameters are required: the initial $w / c$ (here $w / c 0.3$ ), and the hydration degree (calculated from the isothermal calorimetry data and the estimated total heat of hydration, Fig. 2). In summary, there are no input parameters needed, which are not already identified based on other experimental sources. Thus, the model provides blind predictions.

\subsection{Micromechanical analytical model}

The employed formulation and the representation of the multiscale character of cement paste follows the main lines of [17-20] but with no consideration of ITZ. Accordingly, the representation of multiscale nature of cement-based materials (CBM) adopted is shown in supplementary materials [6], see also [18]. The multiscale character of cement-based materials is represented by concrete, mortar, cement paste and hydration product scales. Matrix/inclusions morphology is adopted in all scales. MoriTanaka is used for mortar and concrete scales. Two-coated sphere morphology based on Generalized Self-Consistent scheme is used to represent cement paste scale (i.e. the scale in which the heterogeneities due to the various hydration products can be taken into account). At this scale, the hydrating clinker particle is embedded in a high density (HD) products layer, which is embedded in a low density (LD) products layer.

Evolution of heat of hydration is simulated based only on the composition of cement (in terms of clinker minerals), $w / c$ ratio (see discussion of the influence of $w / c$ of hydration kinetics in [19]) and the particle size distribution following the kinetics model and associated parameters presented in [19].

The estimation of the elastic properties follows [20], but differently of [20] in which the concrete scale effective properties were upscaled through numerical methods, here we use (analytical) MT scheme to upscale concrete effective properties. The evolution of volume fraction of reactants and products are based on:

- hydration kinetics model presented in [19] for which the particle size distribution and its influence in hydration kinetics was explicitly taken into account;

- fitted calorimetry curves associated to calibrations regarding the hydration of individual clinker minerals based on hydration modeling.

In both cases, the kinetics is combined with hydration balance equations. No adjustments parameters are employed (except in the scenario in which (only) calorimetric data were fitted - see Results section comparing both outputs).

\subsection{VCCTL}

Cement hydration was simulated using the Virtual Cement and Concrete Testing Laboratory (VCCTL) model [21], developed at NIST and modified at the Department of Geosciences, University of Padua [22]. The simulated microstructure is used as an input to a FEM module that calculates effective elastic moduli by minimizing the total elastic energy in response to an applied displacement [23]. The intrinsic elastic moduli associated with the different phases are reported elsewhere [22]. Auto-correlation functions are used to spatially distribute the individual clinker phases within the cement particles [24]. The experimental calorimetry curve is used to convert the number of iterations into a physical time scale. The specific surface area, $w / c$ ratio and cement density are supplied as input to a kinetic model [25], which returns the initial number of nuclei and the rate of $\mathrm{C}-\mathrm{S}-\mathrm{H}$ growth, by fitting the time-dependent C-S-H volume obtained by VCCTL, using a gradient descent algorithm. It should be noted that VCCTL implements the core CEMHYD3D functions (see also 3.6) in addition to new implemented functions and modifications. 


\subsection{Micromechanical numerical model}

CEMHYD3D model served for the simulation of cement paste hydration using default parameters [26]. Microstructure was generated at size $100 \times 100 \times 100 \mu \mathrm{m}^{3}$, with the resolution of $1 \mu \mathrm{m} /$ voxel. Cycles to time used standard mapping, which was previously validated on 14 ordinary Portland pastes [27], as:

$$
\text { Time }[h]=3 \cdot 10^{-4} \cdot \text { cycle }^{2}
$$

Volume fraction from CEMHYD3D model provided inputs for a hierarchical micromechanical model. In order to simplify micromechanical simulations, four phases were distinguished, particularly capillary pores, C-S-H, other hydration products and unhydrated cement with gypsum. Entrapped air was assumed as $2 \%$. The hierarchical model uses four scales, particularly C-S-H, C-S-H foam, paste and paste with air. Homogenized properties are passed to higher scales in terms of elastic constants, tensile strength, and fracture energy. This ensures correct energy dissipation within a material volume during crack propagation and gives rise to a deterministic or energetic size effect. Statistical size effect is partially covered by spatial variation of material phases on each scale.

Energetic (non-statistical) size-effect describes material failure by stress redistribution and the associated energy release due to development of large cracks or a large fracture process zone prior to failure. This results to more brittle and macroscopically less strong structures by increasing their sizes [28]. On the other hand, statistical size effect assumes that only the weakest-link determines failure of the whole structure (Weibull theory) [28].

The hierarchical model allows simulating evolution of elastic properties and compressive strength as a function of phase volumes that correspond to each specific degree of hydration [29].

$\mathrm{C}-\mathrm{S}-\mathrm{H}$ globules are assumed to be the weakest solid phase in a heterogeneous multiscale composite. They govern crack propagation and determine macroscopic strength of cement paste. Loading cement paste by compressive stress induces much larger stresses to C-S-H phase and consequently to C$\mathrm{S}-\mathrm{H}$ globules. Such stress magnification origins from stress concentration in heterogeneous microstructures and from the presence of capillary and gel pores. Inverse analysis of a large dataset resulted to identified tensile strength of the globule as $320 \mathrm{MPa}$ [29]. It should be noted that exceeding strength of a single globule leads only to further stress redistribution and damaging significant amount of globules is necessary to reach compressive strength of the paste.

\subsection{HYMOSTRUC3D model}

In 1991, Van Breugel proposed a numerical cement hydration model called HYMOSTRUC [30], the acronym for HYdration, MOrphology and STRUCture formation. The rate of cement hydration was simulated using a function of the water content, temperature, hydration time, etc., of the system. HYMOSTRUC focused on simulating the hydration process of Portland cement. It also simulated the microstructure of cement paste by homogenously distributing the cement particles in a 3D cell. Using this 3D cell microstructure, the contact areas between cement particles were calculated. The calculated contact areas were used to predict the properties of cement-based materials such as compressive strength and elastic properties [31]. Koenders [32] incorporated an algorithm in HYMOSTRUC to simulate the spatial cement particle distribution. Ye [33] incorporated a pixel-based (sampling) algorithm in HYMOSTRUC to analyse the pores of the simulated 3D microstructure. These extensions of HYMOSTRUC [32, 33] concentrated on the 3D microstructure. Hence, the new version of HYMOSTRUC is called HYMOSTRUC3D.

\section{Results and discussion}

In Fig. 2 modelling results of the heat of hydration in the cement paste are presented together with experimental data. A very good agreement could be observed at early hydration stages (up to about 2 d) for model $1-\mathrm{C}-\mathrm{S}-\mathrm{H}$ diffuse filling. Faster initial hydration is predicted with model 1 - C-S-H growing, while better agreement is reached at later stages (after about $1 \mathrm{~d}$ ). On the other hand, hydration heat was overestimated with model 2 at early stages, while better agreement was obtained afterwards. Both model 6 and 7 could reproduce the course of hydration evolution with a very good agreement. It should be mentioned that in the models 1 and 7 the agreement with the experimental data was obtained not as a prediction but by fitting the parameters of the respective models of hydration kinetics to the experimental data presented here. At the same time, the arrangement of the microstructure (hydration products, unhydrated cement and porosity) and the resulting mechanical or transport properties can be simulated with no further fitting. A similar approach is used in the models that simply use experimental data on hydration evolution as an input to the modeling of microstructure, e.g. models 3 and 5.

An interesting case is model 4 in which the parameters of the hydration kinetics models (boundary nucleation and growth kinetics and diffusion-controlled kinetics) were used after [19], where they were fit on another CEM I cement (here denoted as 'Model 4 (no kinetics fitting)'). Even though the initial kinetics agreed well with the experimental data, and hence the modelled cumulative heat curve almost overlapped with the experimental data, a rather poor agreement was obtained in the deceleration phase of hydration kinetics, where the rate of hydration and the cumulative heat were considerably underestimated. The estimations of the model rejoin the experimental curve in the diffusion-driven stage of the model. This shows that even with a simplified assumption regarding hydration kinetics, the predictive capability of the hydration kinetics model can be reasonable at the very early-age (before 1 day) and at late ages (after 5 days). In order to enable further simulations of the elastic properties for the benchmark purposes with the model 4 , the parameters of the hydration 
kinetics model were also fitted (curve denoted as 'Model 4'), leading to a very good agreement with the experimental curve at all ages in Fig. 2.

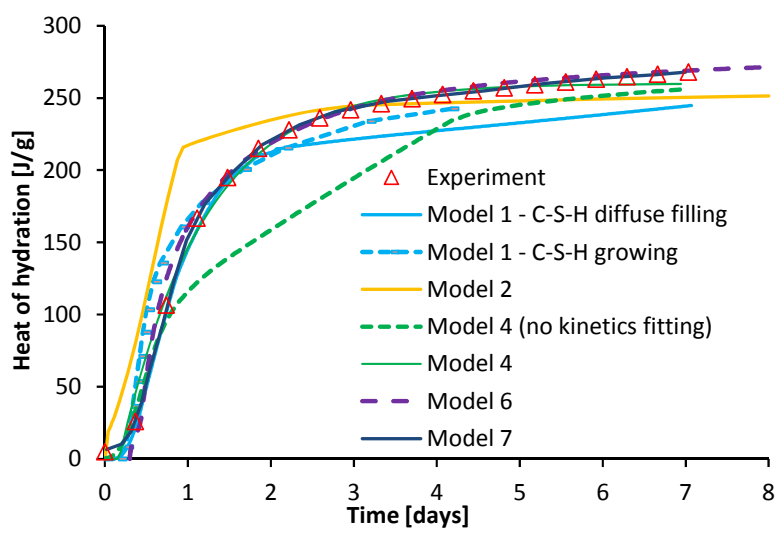

Figure 2. Comparison of the experimental isothermal calorimetry data (average from two replicate samples (difference in cumulative heat of $1 \%$ at most) and simulation results for different models.

In Fig. 3 predictions of the elastic properties (elastic Young's modulus and shear modulus) are presented. It should be noted that the shear modulus was measured experimentally only using the dynamic method, while both static and dynamic methods were used for the elastic Young's modulus. When one considers the dynamic measurements, the trends of the prediction vs. experimental results are similar in the case of elastic Young's modulus and shear modulus. Thus, only the elastic Young's modulus is discussed in detail.

It should be noted here that none of the models used any calibration at the stage of mechanical properties. Instead, for a certain evolution of hydration degree (either fitted to the calorimetry data or simulated with no fitting, see e.g. the two results of model 4), the evolution of the mechanical properties is obtained as resulting from changing volumes and spatial distribution of individual phases and their properties established previously based on direct measurements or taken from the literature. The elastic Young's modulus simulated by model 2 considerably exceeds the measured values and appears to lie outside the range covered by the other models. Before the age of $2 \mathrm{~d}$, this is due to the also overestimated hydration heat release. However, at later ages, the predicted hydration heat evolution agreed well with experimental data, yet the predicted elastic Youngs modulus was considerably overestimated. For the other models, the predicted (or fitted) hydration heat release was similarly in a good agreement with the experimental data, yet the scatter between the different predictions of elastic properties was relatively very high, in particular at early ages, e.g. at $1 d$ the elastic modulus is predicted in the range 6-20 GPa and at $3 \mathrm{~d}$ in the range 14-21 GPa. At later ages, i.e. after about $7 d$ the differences become less significant and the predictions agree relatively well with the experimental data (dynamic elastic Young's modulus), with the spread of the different predictions in the range 19-23 GPa (excluding the model 2 with $31 \mathrm{GPa}$ ). This suggests that the predictive capabilities of the models at early ages, i.e. before about $3 \mathrm{~d}$ should be considered with caution.
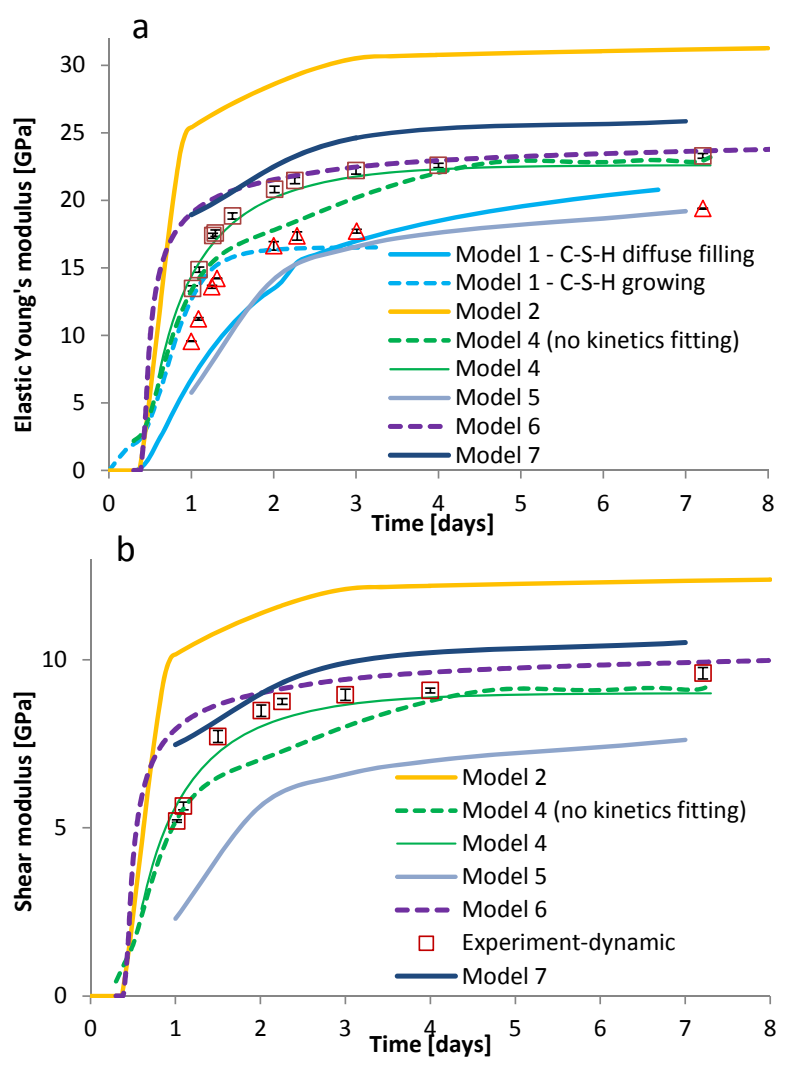

Figure 3. Comparison of the experimental data and simulation results for different models for a) dynamic and static elastic Young's modulus and b) dynamic shear modulus. Experimental results from the dynamic measurements are presented with open square markers and from the static measurements with open triangle markers.

One important issue regards the predictions of the models confronted with either static or dynamic elastic properties. At later ages of about $7 d$, better agreement was found with the experimental data determined using the dynamic method. It has been commonly observed in concrete and porous rocks that the dynamic elastic Young's modulus is higher than the static one, e.g. [4, 22, 34, 35]. The origins of the difference are still under discussion; in general non-linear contributions to the strain [22] or more particular short-term creep [35-37] have been suggested to be responsible for higher deformations measured in the static tests and consequently lower elastic Youngs modulus. It needs to be noted that none of the applied models included explicitly any mechanisms responsible for the lower values of static elastic moduli. Instead, the models used elastic properties of the individual components of the hydrating cement paste. The question thus converges to whether these properties were measured in a way corresponding to dynamic or to static measurements. As discussed in [22], the model predictions based on purely elastic schemes and on the supposedly purely elastic properties determined experimentally should lead to the results close to the dynamic experimental data. On the other hand, the simulations with model 2 refer to the drained conditions and 
the authors state in [12] that an additional estimation of the undrained stiffness is necessary to enable comparison to the dynamic measurements.

In addition to the differences in the modelling schemes being one important origin of the scatter in the predictions between the different models, the used input data (elastic properties of the individual phases) should be considered as another important source of the scatter. The elastic properties of the individual phases used in modelling were obtained as follows. For the anhydrous cement, the data obtained with nanoindentation techniques $[38,39]$ was used in the models. Values for hydrated phases different than the $\mathrm{C}-\mathrm{S}-\mathrm{H}$ were assumed based on nanoindentation techniques with relatively low scatter. On the other hand, for the C-S-H, the majority hydration product by volume, the reported experimental data obtained with nanoindentation is characterized by a large scatter. Model 1 assumes that the Young's modulus of inner C-S-H is constant and uses the value from nanoindentation, but the outer $\mathrm{C}-\mathrm{S}-\mathrm{H}$ is assumed to densify with time. The modulus results are sensitive to the mode of outer C-S-H deposition, with the diffuse filling model yielding results which are closer to the experiment at later ages. Model 2 uses a Young's modulus for the solid part of both inner and outer C-S-H gels determined by inverse analysis in [12] from nanoindentation tests on inner C-S-H gel reported in [40]. This is required, as the micromechanical model [12] considers inner C-S-H gel to be of a constant porosity, while outer C-S-H gel porosity decreases as hydration proceeds. Models 4 and 6 used nanoindentation data for the so-called low and high-density (LD and HD) C-S$\mathrm{H}$ published in $[41,42]$. Model 5 used a simple homogenization scheme for calculating the stiffness of the "saturated C-S-H" [22] and estimated values close to the nanoindentation data [40] used also in the other models. In model 7 , the compressive strength and the dynamic moduli of cement pastes were directly related to the degree of interparticle bonding (connectivity) of the solid phase in the microstructure of cementitious materials [31]. An interesting observation regards the fact that when the same set of input data on elastic properties of individual phases is used, the models can provide rather similar predictions, compare models 4 and 6 in Fig. 3 . At the same time, even if similar nanoindentation results are used, the predictions are still strongly affected by the assumed homogenization scheme for the elastic properties of the $\mathrm{C}-\mathrm{S}-\mathrm{H}$.

In Fig. 4, predictions of compressive strength are compared with the experimental data. A very good agreement was found with model 3 . It is worth mentioning that in this case the only input parameters were water-to-cement ratio (governing the initial volumetric composition) and evolution of hydration degree. This input can be also used in a very simple approach based on gel/space ratio proposed by Powers [43], see also [26]. In this approach, compressive strength $f_{c}$ is calculated as:

$$
f_{c}=f_{c 0}(\text { gel } / \text { space })^{n}
$$

where $n$ is assumed as equal to 2.6 after [26], $f c_{0}$ is intrinsic strength (141.5 MPa) obtained by fitting the strength to the value at $3 \mathrm{~d}$, and gel-space ratiois calculated as:

gel $/$ space $=0.68 \alpha /(0.32 \alpha+w / c$

where $\alpha$ is hydration degree. It can be readily seen that the prediction with the Powers model agree very well with those from model 3 (and with experimental data).

By using interparticle connectivity of the solid phase, model 7 shows also a good agreement with the experiments. For model 6 , the predicted compressive strength overestimates the measured values at later stages. This could be related to model calibration, which used often smaller cylinder samples for calibration, compared with tested prisms of $25 \times 25 \times 100$ $\mathrm{mm}^{3}$.

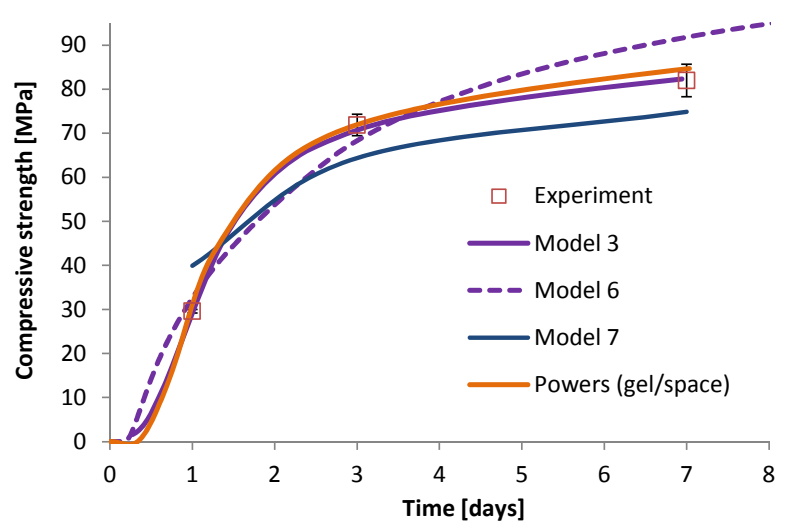

Figure 4. Comparison of the experimental data and simulation results for compressive strength.

\section{Conclusions}

The results of the numerical benchmark on modelling evolution of the microstructure of cement paste have been presented. The benchmark was based on an experimental dataset published previously, i.e. both the input data (material properties, mix compositions etc.) and the experimental results were open to the participants. The part of the benchmark presented here focused on modeling a $w / c 0.30$ cement paste hydrating in sealed conditions. 7 groups applying different analytical and numerical models of the evolution of microstructure and capable of simulating the evolution of concrete properties took part in the campaign. In this paper, a part of the results was presented comprising evolution of hydration heat release and mechanical properties (elastic Young's modulus, shear modulus and compressive strength). As long as the evolution of hydration degree (estimated based on the hydration heat release) is in many models used directly as input data or fitted, the mechanical properties were simulated with no fitting, based only on previously established experimental input data. In all the models presented here, modelling of mechanical properties was carried out based on an established database of the mechanical properties of the individual phases of a hydrating cement paste. 
A considerable scatter between the predictions delivered by the different models could be observed, in particular at early ages before about $3 \mathrm{~d}$ (e.g. predicted Young's modulus at $1 \mathrm{~d}$ in the range 6-20 GPa), while lower spread of the predictions and better agreement with experimental data on dynamic Young's modulus was found at later ages, e.g. at $7 \mathrm{~d}$. The scatter in the predictions is both due to the different modelling schemes applied and due to the scatter in the input data, in particular elastic properties of the individual phases of the hydrating cement paste used in the different models. The latter is especially due to the large scatter of the data on C-S-H elastic properties obtained from nanoindentation measurements. This could represent a considerable source of error, since $\mathrm{C}-\mathrm{S}-\mathrm{H}$ represents the most abundant phase in hydrated (Portland) cement pastes and likely exerts a strong control on the effective elastic properties of the material. The scatter is mainly due to the the short-range order of the $\mathrm{C}-\mathrm{S}-\mathrm{H}$ and therefore the indented regions encompassing different solid phases or porosity with the corresponding overlapping mechanical properties $[44,45]$. Another reason may be due to probing $\mathrm{C}-\mathrm{S}-\mathrm{H}$ domains having different orientations. It has to be noted, in fact, that solids having some degree of ordering tend to be elastically anisotropic (i.e. elastic properties depend on spatial orientation) [46].

Only three models were applied for prediction of compressive strength providing good agreement with the experimental data.

The results of this benchmark campaign suggest that the predictions of the models should be always treated with caution. Even though each of the models applied here had been previously validated with chosen sets of experimental data, the example presented here shows that the large scatter can arise even for a simple system of an ordinary cement paste.

As long as some models provided quite accurate predictions, it should be noted that only one material was studied in this benchmark and further studied are necessary to confirm the predictive capacities. In fact, the presented benchmark was a preliminary attempt to recognize the demand in case of specific input necessary for different models and demonstrate the output that these models can provide. A broader benchmarking study with larger experimental data obtained from the 'Extended Round Robin Testing Program' RRT+ [2] is currently in course as an activity of the COST Action TU1404.

\section{Acknowledgements}

The benchmark presented in this paper was carried out within a framework of activities of work group 2 of the COST Action TU1404 "Towards the next generation of standards for service life of cement-based materials and structures". Financial support of FEDER/COMPETE2020/FCT through POCI-01-0145-FEDER-007633 and PTDC/ECMEST/1056/2014 (POCl-01-0145-FEDER-016841) is also acknowledged.

\section{References}

[1] COST, Document 061/14. Memorandum of Understanding for the implementation of a European Concerted Research Action designated as COST Action TU1404: Towards the next generation of standards for service life of cement-based materials and strutcures. COST - European Cooperation in the field of Scientific and Technical Research, 2014.

[2] M. Serdar, et al., COST Action TU1404: recent advances of the Extended Round Robin Test RRT+. 2nd International RILEM/COST Conference on Early Age Cracking and Serviceability in Cement-Based Materials and Structures, 2017.

[3] M. Wyrzykowski, P. Lura, Controlling the coefficient of therma expansion of cementitious materials - A new application for superabsorbent polymers. Cem Concr Compos (2013) 35: 4958. https://doi.org/10.1016/j.cemconcomp.2012.08.010

[4] M. Wyrzykowski, P. Lura, Moisture dependence of thermal expansion in cement-based materials at early ages. Cem Concr Res (2013) 53: 25-35.

https://doi.org/10.1016/j.cemconres.2013.05.016

[5] H.F.W. Taylor, Modification of the Bogue calculation. Advances in Cement Research (1989) 2: 73-77.

https://doi.org/10.1680/adcr.1989.2.6.73

[6] M. Wyrzykowski, et al., Numerical benchmark campaign of COST Action TU1404 - microstructural modelling supplementary materials. RILEM Technical Letters (2017) 2.

[7] S. Bishnoi, K.L. Scrivener, $\mu$ ic: A new platform for modelling the hydration of cements. Cem Concr Res (2009) 39: 266-274. https://doi.org/10.1016/i.cemconres.2008.12.002

[8] C. Dunant, et al., Architecture tradeoffs of integrating a mesh generator to partition of unity enriched object-oriented finite element software. European Journal of Computational Mechanics/Revue Européenne de Mécanique Numérique (2007) 16: 237-258.

[9] C. Dunant, E. Bentz, Algorithmically imposed thermodynamic compliance for material models in mechanical simulations using the AIM method. International Journal for Numerical Methods in Engineering (2015) 104: 963-982. https://doi.org/10.1002/nme.4969

[10] H.Q. Do, S. Bishnoi, K.L. Scrivener, Microstructural modelling of the elastic properties of tricalcium silicate pastes at early ages. Computers And Concrete (2015) 16: 125-140. https://doi.org/10.12989/cac.2015.16.1.125

[11] J. Sanahuja, N.-C. Tran, L. Charpin, L. Petit, Material properties prediction for long term operation of nuclear power plants civil engineering structures: challenges at EDF. 1st International Conference on Grand Challenges in Construction Materials, Los Angeles, USA, 2016, pp. 12.

[12] J. Sanahuja, L. Dormieux, G. Chanvillard, Modelling elasticity of a hydrating cement paste. Cem Concr Res (2007) 37: 14271439. https://doi.org/10.1016/j.cemconres.2007.07.003

[13] L. Charpin, et al., Multiscale modeling of hydration, elasticity and creep of VeRCoRs concrete. Focus on creep characteritic times. . TINCE 3, Paris, France, 2016.

[14] B. Pichler, C. Hellmich, Upscaling quasi-brittle strength of cement paste and mortar: A multi-scale engineering mechanics model. Cem Concr Res (2011) 41: 467-476. https://doi.org/10.1016/i.cemconres.2011.01.010

[15] B. Pichler, et al., Effect of gel-space ratio and microstructure on strength of hydrating cementitious materials: An engineering micromechanics approach. Cem Concr Res (2013) 45: 55-68. https://doi.org/10.1016/i.cemconres.2012.10.019

[16] G. Constantinides, F.-J. Ulm, The nanogranular nature of C-S-H. Journal of the Mechanics and Physics of Solids (2007) 55: 64-90. https://doi.org/10.1016/i.jmps.2006.06.003

[17] T. Honorio, B. Bary, F. Benboudjema, Estimation of elastic properties of cement based materials at early age based on a combined numerical and analytical multiscale micromechanics approach. RILEM Int. Symp. Concr. Model., Beijing, China, 2014.

[18] T. Honorio, B. Bary, F. Benboudjema, Multiscale estimation of the thermal properties of cement-based materials at early-age. SSCS, 2015

[19] T. Honorio, B. Bary, F. Benboudjema, S. Poyet, Modeling hydration kinetics based on boundary nucleation and spacefilling growth in a fixed confined zone. Cem Concr Res (2016) 83: 31-44. https://doi.org/10.1016/j.cemconres.2016.01.012 
[20] T.H. de Faria, Modelling Concrete Behaviour At Early-Age: Multiscale Analysis And Simulation Of A Massive Disposal Structure. École normale supérieure de Cachan-ENS Cachan, 2015.

[21] D.P. Bentz, et al., Virtual testing of cement and concrete. Significance of tests and properties of concrete and concretemaking materials. ASTM International2006.

[22] L. Valentini, et al., Simulation of the hydration kinetics and elastic moduli of cement mortars by microstructural modelling. Cem Concr Compos (2014) 52: 54-63. https://doi.org/10.1016/i.cemconcomp.2014.05.005

[23] E.J. Garboczi, A. Day, An algorithm for computing the effective linear elastic properties of heterogeneous materials: threedimensional results for composites with equal phase Poisson ratios. Journal of the Mechanics and Physics of Solids (1995) 43: 1349-1362. https://doi.org/10.1016/0022-5096(95)00050-S

[24] D.P. Bentz, Quantitative comparison of real and CEMHYD3D model microstructures using correlation functions. Cem Concr Res (2006) 36: 259-263.

https://doi.org/10.1016/j.cemconres.2005.07.003

[25] L. Valentini, et al., Kinetic model of calcium-silicate hydrate nucleation and growth in the presence of PCE superplasticizers. Crystal Growth \& Design (2016) 16: 646-654. https://doi.org/10.1021/acs.cgd.5b01127

[26] D.P. Bentz, CEMHYD3D: A three-dimensional cement hydration and microstructure development modelling package. Version 2.0. National Institute of Standards and Technology Interagency Report (2000) 7232

[27] V. Smilauer, Multiscale Hierarchical Modeling of Hydrating Concrete. Saxe-Coburg Publications, 2015.

[28] Z.P. Bazant, J. Planas, Fracture and size effect in concrete and other quasibrittle materials. CRC press, 1997.

[29] M. Hlobil, V. Šmilauer, G. Chanvillard, Micromechanical multiscale fracture model for compressive strength of blended cement pastes. Cem Concr Res (2016) 83: 188-202. https://doi.org/10.1016/j.cemconres.2015.12.003

[30] K. Van Breugel, Simulation of hydration and formation of structure in hardening cement-based materials. PhD Thesis, Delft, Delft University of Technology, The Netherlands, 1991.

[31] Z. Sun, G. Ye, S.P. Shah, Microstructure and early-age properties of Portland cement paste-effects of connectivity of solid phases. ACI Materials Journal (2005) 102: 122-129.

[32] E.A.B. Koenders, Simulation of volume changes in hardening cement-based materials. PhD Thesis, Delft University of Technology, Delft, The Netherlands, 1997.

[33] G. Ye, Experimental Study and Numerical Simulation of the Development of the microstructure and permeability of cementitious materials. PhD Thesis, Delft, Delft University of Technology, The Netherlands, 2003.

[34] S. Kolias, R.I.T. Williams, Relationships between the static and the dynamic moduli of elasticity in cement stabilised materials. Mater Struct (1980) 13: 99-107. https://doi.org/10.1007/BF02473806

[35] D. Orchard, J. Walker, D. Stewart, A study of the relationship between the static and dynamic modulus of elasticity and other properties of certain materials. Australian Road Research Board (ARRB) Conference, 3rd, 1966, Sydney, 1966.

[36] M. Wyrzykowski, P. Lura, The effect of external load on internal relative humidity in concrete. Cem Concr Res (2014) 65: 58-63. https://doi.org/10.1016/j.cemconres.2014.07.011

[37] M. Irfan-ul-Hassan, B. Pichler, R. Reihsner, C. Hellmich, Elastic and creep properties of young cement paste, as determined from hourly repeated minute-long quasi-static tests. Cem Concr Res (2016) 82: 36-49. https://doi.org/10.1016/j.cemconres.2015.11.007

[38] K. Velez, et al., Determination by nanoindentation of elastic modulus and hardness of pure constituents of Portland cement clinker. Cem Concr Res (2001) 31: 555-561. https://doi.org/10.1016/S0008-8846(00)00505-6

[39] P. Acker, Micromechanical analysis of creep and shrinkage mechanisms. Creep, Shrinkage and Durability Mechanics of Concrete and other quasi-brittle Materials, Cambridge, MA (2001) 15-25.

[40] P. Acker, F.-J. Ulm, Creep and shrinkage of concrete: physical origins and practical measurements. Nuclear Engineering and Design (2001) 203: 143-158.

https://doi.org/10.1016/S0029-5493(00)00304-6
[41] O. Bernard, F.-J. Ulm, E. Lemarchand, A multiscale micromechanics-hydration model for the early-age elastic properties of cement-based materials. Cem Concr Res (2003) 33: 1293-1309.

$$
\text { https://doi.org/10.1016/S0008-8846(03)00039-5 }
$$

[42] G. Constantinides, F.-J. Ulm, The effect of two types of C-S-H on the elasticity of cement-based materials: Results from nanoindentation and micromechanical modeling. Cem Concr $\operatorname{Res}(2004)$ 34: 67-80. https://doi.org/10.1016/S0008-8846(03)00230-8

[43] T.C. Powers, T.L. Brownyard, Studies of the physical properties of hardened Portland cement paste. ACl Journal Proceedings. ACl, 1946.

[44] W. Wilson, L. Sorelli, A. Tagnit-Hamou, Automated coupling of Nanolndentation and Quantitative Energy-Dispersive Spectroscopy (NI-QEDS): A comprehensive method to disclose the micro-chemo-mechanical properties of cement pastes. Cem Concr Res (2017).

[45] P. Trtik, B. Münch, P. Lura, A critical examination of statistical nanoindentation on model materials and hardened cement pastes based on virtual experiments. Cem Concr Compos (2009) 31: 705-714 https://doi.org/10.1016/i.cemconcomp.2009.07.001

[46] [46] R.J. Angel, J.M. Jackson, H.J. Reichmann, S. Speziale, Elasticity measurements on minerals: a review. European Journal of Mineralogy (2009) 21: 525-550. https://doi.org/10.1127/0935-1221/2009/0021-1925 